\title{
Effects of GI Meals on Intermittent Exercise
}

Authors

Affiliation

\section{A. T. Hulton, W. Gregson, D. Maclaren, D. A. Doran}

Liverpool John Moores University, Research Institute for Sport \& Exercise Sciences, Liverpool, United Kingdom
Key words

- pre-exercise nutrition

- carbohydrates

- glycaemic index

- football-simulated exercise

accepted after revision

December 05, 2011

\section{Bibliography}

DOI http://dx.doi.org/

10.1055/s-0031-1299754

Published online:

June 15, 2012

Int I Sports Med 2012; 33:

756-762 @ Georg Thieme

Verlag KG Stuttgart · New York

ISSN 0172-4622

\section{Correspondence}

Andrew Thomas Hulton

Liverpool John Moores

University

Sport \& Exercise Sciences

Tom Reilly Building

L3 3AF Liverpool

United Kingdom

Tel.: +44/0151/2314067

Fax: +44/0151/231 4353

a.t.hulton@ljmu.ac.uk

\section{Abstract \\ $\nabla$}

Pre-exercise meals or single foods containing low glycaemic index (LGI) carbohydrates (CHO) have been shown to enhance performance prior to prolonged steady state exercise compared to high glycaemic index (HGI) CHO. This study investigated the impact of HGI and LGI preexercise meals on intermittent high intensity exercise. Nine male recreational football players performed a football specific protocol followed by a $1 \mathrm{~km}$ time trial $3.5 \mathrm{~h}$ after ingesting 1 of 2 isoenergetic test meals (HGI: $870.3 \mathrm{kcal}$, LGI: $889.5 \mathrm{kcal}$ ), which were either HGI (GI: 80) or LGI (GI: 44). Blood glucose, fatty acids (FA),

\section{Introduction}

$\nabla$

A primary cause of fatigue in football and similar intermittent sports is the depletion of muscle glycogen. Previous research [26] has observed both a significant reduction in muscle glycogen after a football match, and also that distance covered in the second half was reduced for players with a lower starting level of muscle glycogen compared to those with a higher level. The need for high carbohydrate ( $\mathrm{CHO}$ ) diets prior to prolonged intermittent exercise was reinforced [1] by demonstrating a greater exercise capacity following a $48 \mathrm{~h}$ high $\mathrm{CHO}$ diet (65\% energy intake from $\mathrm{CHO}$ ) compared to a control diet (39\% energy intake from $\mathrm{CHO}$ ). There is also evidence that carbohydrate feeding prior to an activity has a significant impact on subsequent performance $[23,24]$ either by enhancing muscle glycogen stores or ensuring availability of blood glucose in the later stages of exercise. Taken together, these studies highlight the importance of ingesting sufficient $\mathrm{CHO}$ to maintain adequate levels of muscle glycogen prior to an intermittent field sport activity such as football. glycerol, $\beta$-hydroxybutyrate, lactate and insulin were assessed before, during, and after the exercise bout, whilst rates of $\mathrm{CHO}$ and fat oxidation were determined at 4 time points during the protocol. No significant differences were found for the $1 \mathrm{~km}$ time trial (LGI: $210.2 \pm 19.1 \mathrm{~s}$ : HGI: $215.8 \pm 22.6 \mathrm{~s})($ mean $\pm \mathrm{SD})$, nor for any of the other variables measured $(P>0.05)$ apart from a significant condition effect with FA and significant interaction effects observed for glucose, $\beta$-hydroxybutyrate and lactate $(\mathrm{P}<0.05)$. These findings suggest that the type of $\mathrm{CHO}$ ingested in a pre-match meal has no significant impact on performance or metabolic responses during $90 \mathrm{~min}$ of intermittent high intensity exercise.
Carbohydrate foods may be categorised by their ability to increase blood glucose; a concept which has resulted in the term glycaemic index (GI). The GI was initially developed to enable healthcare professionals to prescribe diets of low glycaemic index (LGI) or high glycaemic index (HGI) foods to assist blood glucose control in diabetics [16]. In a sport and exercise context, the GI has been used to investigate the influence of preexercise carbohydrate intake on subsequent performance and metabolism.The application of GI for sport was first studied in 1991 [32] and concluded that a LGI food (i.e. lentils) produced a greater endurance capacity compared to a HGI food (i.e. potatoes) fed $1 \mathrm{~h}$ before cycling to exhaustion at $65-70 \% \mathrm{VO}_{2 \max }$. Subsequently, it has been found that a LGI meal improved $16 \mathrm{~km}$ time trial performance following a $5 \mathrm{~km}$ run at $70 \% \mathrm{VO}_{2 \max }[35]$, whilst others [8] have observed a significant increase in time to fatigue at $100 \%$ $\mathrm{VO}_{2 \max }$ after cycling $2 \mathrm{~h}$ at $70 \% \mathrm{VO}_{2 \max }$ when fed a LGI meal. Recently, a study investigating the effects of GI on football performance, measured by total distance covered during 5 sprints throughout the last $15 \mathrm{~min}$ of a football protocol, 
Table 1 Energy, macronutrient content and Gl of the test meals.

\begin{tabular}{|c|c|c|c|c|c|c|}
\hline Food & Amount & Carbohydrate (g) & Protein (g) & Fat (g) & Energy (kcals) & GI \\
\hline \multicolumn{7}{|l|}{ High GI meal } \\
\hline lucozade original & $380 \mathrm{ml}$ & 65.4 & 0 & 0 & 245.3 & 95 \\
\hline water & $210 \mathrm{ml}$ & - & - & - & - & - \\
\hline askash rice & $63 \mathrm{~g}$ & 50 & 4.5 & 0.1 & 206.4 & 78 \\
\hline chicken breast & $100 \mathrm{~g}$ & 0.3 & 26.1 & 5.8 & 157.7 & 0 \\
\hline tomato based sauce & $300 \mathrm{~g}$ & 23.1 & 5.1 & 17.1 & 260.9 & 49 \\
\hline total & - & 138.8 & 35.7 & 23 & 870.3 & 80 \\
\hline \multicolumn{7}{|l|}{ Low GI meal } \\
\hline apple juice & $590 \mathrm{ml}$ & 65.5 & 0.6 & 0 & 248.0 & 40 \\
\hline basmati rice & $63 \mathrm{~g}$ & 44.8 & 6.1 & 0.8 & 199.6 & 48 \\
\hline chicken breast & $100 \mathrm{~g}$ & 0.3 & 26.1 & 5.8 & 157.7 & 0 \\
\hline tomato based sauce & $300 \mathrm{~g}$ & 23.1 & 5.1 & 17.1 & 260.9 & 49 \\
\hline total & - & 133.7 & 37.9 & 23.7 & 866.3 & 44 \\
\hline
\end{tabular}

found no performance benefits between meals of varying GI [19]. However, the meals used in this study did not reflect that of current practise within sport, as only one item of food was administered (low GI: boiled red lentils. High GI: instant mash potato with egg whites and ketchup). Other studies have found no effects on performance with LGI pre-exercise meals, although they have observed a metabolic shift to an increase in fat oxidation and a lower insulinaemic response [10,11,27]. The equivocal results are possibly due to variations in exercise duration and intensity, test meal composition, and the timing of ingestion. Furthermore, many of the studies comparing high and low GI meals $[8,10,20,27,28]$ fail to offer complete meals typical of pretraining or pre-competition. In addition the timing of the meals often does not replicate that employed in elite sport, where athletes would typically eat meals $3-4 \mathrm{~h}$ prior to competition.

This investigation compared a HGI and a LGI meal fed to participants $3.5 \mathrm{~h}$ prior to $90 \mathrm{~min}$ of intermittent high intensity exercise. This approach conveys a realistic scenario commonly observed in the professional game as rounded meals rather than individual food components are consumed within this prematch time frame. We hypothesised that the LGI meal would promote fat availability and oxidation, maintain greater glucose stability in the postprandial period, and result in enhanced $1 \mathrm{~km}$ running performance after $90 \mathrm{~min}$ of intermittent high intensity exercise.

\section{Method \\ $\nabla$}

\section{Participants}

Nine male recreational football players, all of whom trained twice a week and played a weekly competitive match were recruited for the study (Age: $21 \pm 3$ years, body mass [BM]: $74.4 \pm 4.4 \mathrm{~kg}$, body height $180 \pm 8 \mathrm{~cm}$ ). Participants provided written informed consent and the study was approved by the University Ethics Committee. Furthermore, the study was performed in accordance with the ethical standards of the International Journal of Sports Medicine [15].

\section{Experimental design}

Each participant was required to attend the laboratory on a minimum of 4 separate occasions. The first 2 were for familiarisation of the intermittent treadmill protocol and the $1 \mathrm{~km}$ performance test. Familiarisation of the $1 \mathrm{~km}$ performance test was achieved when test-retest times were within $2 \%$. The final 2 visits were to complete the experimental conditions, which were conducted in a counterbalanced, randomized manner separated by 7 days.

\section{Test meals}

Test meals were matched for macronutrient content and fluid. The size of test meals represented approximately $2 \mathrm{~g} / \mathrm{kg}$ BM for a $70 \mathrm{~kg}$ participant. This amount was used as meals of greater quantity were deemed too large to consume. Participant's diet in the $24 \mathrm{~h}$ period prior to the first condition was recorded in a food diary, the subsequent trial was undertaken following the participant's verbal confirmation that the previous diet was followed. On the morning of both conditions, participants consumed the same standardised breakfast, of cereal, toast and fruit juice (total energy $=377 \mathrm{kcal} ; \mathrm{CHO}=79 \mathrm{~g}$; Fat $=3 \mathrm{~g}$; Protein $=13 \mathrm{~g}$ ), which was consumed at 08:00. Participants abstained from alcohol and any physical exercise for $48 \mathrm{~h}$ prior to the test. $\odot$ Table 1 provides macronutrient details of the meals with their GI value and energy content. GI values were derived from previous reports [12] and the GI of the meal was calculated using the mixed-meal method [34].

\section{Protocol}

Following collection of the first venous blood sample $(20 \mathrm{~mL})$ at approximately $11: 10$, participants consumed the test meal at 11:30 (i.e. $3.5 \mathrm{~h}$ before the start of exercise which replicates the current practice of professional footballers). Blood glucose samples (Glucose Hemocue 201+, Hemocue, Ängelholm, Sweden) were also collected from finger prick samples pre-meal, and at 5, 30 , and 60 min post meal. During the postprandial period, participants drank $1 \mathrm{~L}$ of water and rested until they returned to the laboratory at 14:30 for the pre-exercise blood sample.

Participants completed a standardised warm up consisting of light jogging and static stretching prior to the start of the protocol at 15:00. The football specific protocol was an amended football protocol [9] completed on a motorised treadmill $(\mathrm{H} / \mathrm{P}$ Cosmos Pulsar, Nussdorf-Traunstein, Germany). This protocol has been used in a previous study investigating hydration strategies for football [7]. The protocol imitates the activity patterns that occur in football matches and include bouts of walking (31$39 \mathrm{~s})$, jogging (41-46s), cruising (41-45s), sprinting (16-21s) and standing. The proportion of each individual activity is similar to those obtained from motion analysis [25]. The protocol comprises of two $22 \mathrm{~min} 30$ s blocks to comprise one half of $45 \mathrm{~min}$; a total of 4 blocks with a $15 \mathrm{~min}$ half time constituted the whole trial. 


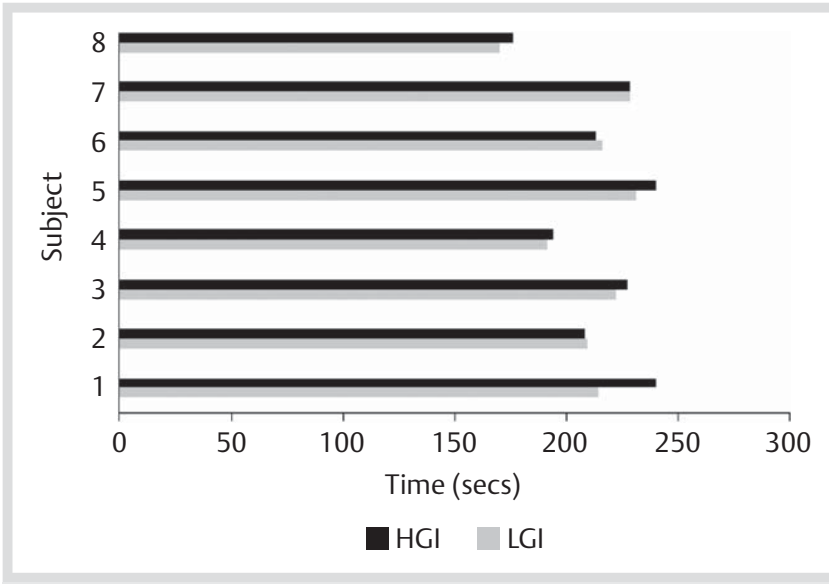

Fig. 1 Time trial performance.

Blood samples were collected after 10, 20 and 30 min into the first half of the protocol and immediately analysed for blood glucose to check for evidence of hypoglycaemia. A 15 min half time break was provided during which subjects drank $5 \mathrm{~mL} \cdot \mathrm{kg}^{-1}$ of water and a further venous blood sample was collected. The second $45 \mathrm{~min}$ was a replication of activity patterns experienced during the first $45 \mathrm{~min}$, totalling $90 \mathrm{~min}$. A final venous blood sample was collected immediately post-exercise before participants ran the $1 \mathrm{~km}$ performance test.

The performance test was a self-paced $1 \mathrm{~km}$ time trial during which participants controlled the treadmill speed but were blinded to the actual speeds and time displayed on the treadmill. A performance test rather than a test for exercise capacity (i.e., time to fatigue) was deemed more appropriate and represented a consideration of 'what was left in the fuel tank'. Furthermore, such a high intensity task would necessitate use of all muscle fibres, although the type IIx fibres would be significantly employed [14].

At 4 time points during the protocol $(10,35,55$, and $80 \mathrm{~min})$ expired gas was measured (Cortex Metamax online gas analyser, Cortex, Leipzig, Germany) from which calculations of fat and $\mathrm{CHO}$ oxidation were made [13]. The expired gas collections were collected in a similar fashion to previous authors [1], during which the measures were collected for 5 min during a range of exercise intensities but ending with a low intensity bout. In addition, the first sample was not collected until after $10 \mathrm{~min}$, which should ensure $\mathrm{CO}_{2}$ metabolism was in a steady state.

Subjective measurements of rating of perceived exertion [5] were recorded every $5 \mathrm{~min}$, while hunger and gut fullness scales [31] were recorded every $15 \mathrm{~min}$. Heart rate was continually measured and averaged over 5 min (Polar S610, Polar, Kempele, Finland).

Venous blood samples were collected from the antecubital vein pre-meal, pre-exercise, at half time, and post exercise, and analysed for fatty acid (FA), glycerol, $\beta$-hydroxybutyrate and lactic acid (RX Daytona clinical chemistry analyser, Randox, Co. Antrim, UK). Serum samples were collected following the same method as described above, for the analysis of insulin (Immulite 1000 immunoassay analyser, Siemens Healthcare Diagnostics, Illinois, USA). Blood glucose samples (Glucose Hemocue 201+, Hemocue, Ängelholm, Sweden) were collected at the same time points, but analysed separately to ensure a continual analysis from capillary blood. Intra assay coefficient of variation was
$2.27 \%$ for glucose, $3.71 \%$ for FA, $0.60 \%$ for glycerol, $5.33 \%$ for $\beta$-hydroxybutyrate, $2.15 \%$ for lactic acid and $3.54 \%$ for insulin.

\section{Statistics}

SPSS software (version 17 SPSS, Chicago, IL) was used for data entry and analysis. Analysis of variance for repeated measures on 2 factors (experimental condition and time) was used to analyse differences in the physiologic and metabolic responses in both trials. If a significant interaction was obtained, a least significance difference post hoc test was used to determine the location of the variance [4]. A paired t-test was used to analyse time trial performance times. Differences were considered significant at $\mathrm{P}<0.05$. All data is presented as the mean $\pm \mathrm{SD}$.

\section{Results \\ $\nabla$}

\section{Performance}

One of the participants was unable to complete one of the timed runs due to a musculoskeletal injury and so the data are based on $\mathrm{n}=8$. No significant difference was found between conditions for the $1 \mathrm{~km}$ time trial $(P>0.05)$. Completion times were $210.2 \pm 19.1 \mathrm{~s}$ for LGI and $215.8 \pm 22.6 \mathrm{~s}$ for the HGI which represented a $5.6 \mathrm{~s}$ (or $2.78 \%$ ) faster completion after LGI. Furthermore, 5 out of 8 participants were faster after LGI, 2 were faster following HGI, and 1 produced identical results ( 0 Fig. 1 ).

\section{Blood glucose prior to exercise}

No significant differences were observed between conditions, but significant differences were seen for time $(P=0.008)$ and for an interaction $(P=0.050)$. For both conditions the blood glucose concentration increased following consumption of the test meals with the HGI meal producing a greater increase from $4.6 \mathrm{mmol} . \mathrm{L}^{-1}$ to $7.2 \mathrm{mmol}^{-1}$ compared to $4.9 \mathrm{mmol} . \mathrm{L}^{-1}$ to $5.7 \mathrm{mmol}^{-\mathrm{L}^{-1}}$ after LGI. Blood glucose values subsequently decreased throughout the postprandial period with HGI resulting in a mean value of $4.0 \mathrm{mmol}^{-1} \mathrm{~L}^{-1}$ prior to the start of exercise, whereas the blood glucose response for LGI remained more constant following the initial increase, resulting in a mean value of $4.8 \mathrm{mmol. \textrm {L } ^ { - 1 }}$ prior to the start of exercise ( 0 Fig. 2 ).

\section{Blood glucose during exercise}

No significant differences were observed between conditions, time or an interaction during exercise $(\mathrm{P}>0.05)$. Blood glucose remained similar throughout exercise for both conditions (o Fig. 2).

\section{Serum insulin}

Serum insulin concentration increased for both conditions following the test meals, with no significant differences evident between conditions, time or interactions $(\mathrm{P}>0.05)$ ( $\bullet$ Fig. 2 ).

\section{Plasma metabolites}

No significant difference between conditions or interactions was seen for glycerol $(P>0.05)$, although a significant effect of time was observed $(P=0.001)$ as glycerol concentrations increased throughout exercise. Significant differences were observed between conditions for $F A(P=0.022)$ along with a significant time effect $(\mathrm{P}=0.001)$, but no interaction effect was observed $(P>0.05)$. FA concentrations remained similar following consumption of the tests meals, but LGI resulted in a greater increase 


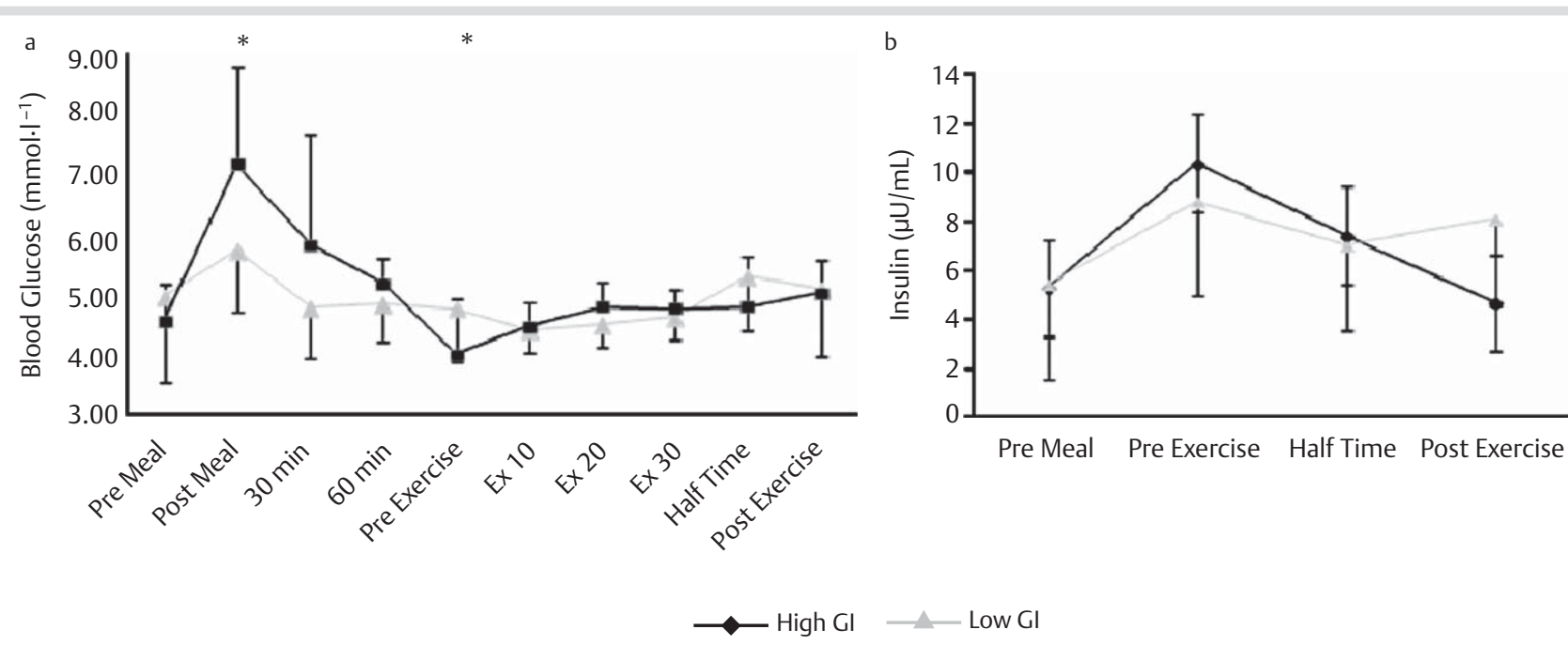

Fig. 2 a Blood glucose concentration prior to the test meal and throughout the postprandial period and subsequent protocol. b Insulin concentration prior to the test meal and throughout exercise.

* significant interactions occur

in FA rising from $0.10 \mathrm{mmol} \cdot \mathrm{L}^{-1}$ at the onset of exercise to $1.70 \mathrm{mmol} . \mathrm{L}^{-1}$ post exercise, compared to $0.07-1.33 \mathrm{mmol} . \mathrm{L}^{-1}$ for HGI. Significant time $(P=0.001)$ and interaction $(P=0.001)$ effects were observed for $\beta$-hydroxybutyrate with a post exercise result of $0.106 \mathrm{mmol} . \mathrm{L}^{-1}$ for LGI compared to $0.058 \mathrm{mmol} . \mathrm{L}^{-1}$ for the HGI. Lactate concentrations increased from rest throughout exercise, with only a slight decrease from half time to post exercise in HGI. No significant differences were found between conditions, but a significant difference over time was observed $(P=0.002)$, as was a significant interaction $(P=0.020)$ ( $\bullet$ Fig. 3 ).

\section{Rate of fat and $\mathrm{CHO}$ oxidation}

Rates of $\mathrm{CHO}$ and fat oxidation were similar throughout both conditions. No significant condition or interaction effects were observed, but a time effect was seen for both $\mathrm{CHO}(\mathrm{P}=0.012)$ and fat $(\mathrm{P}=0.013)$ ( $\bullet$ Fig. 4).

Heart Rate and subjective rating scales $\mathrm{HR}$ and RPE both increased significantly over time $(P=0.001)$, but there was no significant difference between conditions. Subjective ratings of fullness and hunger showed no significant difference between conditions, over time or an interaction $(\mathrm{P}>0.05)(\bullet$ Fig. 5$)$.

\section{Discussion \\ $\nabla$}

The main finding from this study was that consuming a LGI or HGI meal concordant with a time mirroring current practice in professional (and most recreational) football, and prior to intermittent high intensity exercise, had no significant effect on performance or metabolic responses with our recreational football players. These findings are in support of investigations that have observed no significant changes in fat oxidation [35], fat metabolites [22,29], or performance [10,11,19,20,27,33] following a LGI food/meal. In contrast some studies have observed enhanced fat oxidation [30,33], increased FA or glycerol or ketone body concentrations $[10,11,27,30]$, and even performance $[8,22,32$, 35] with LGI foods. The difference accruing from these investigations is that they either invariably use single foods $[10,11,27]$ or modes of exercise such as prolonged steady state cycling
$[10,11,22,27]$ or prolonged steady state running [36]. However, a recent study explored the effect of GI meals on prolonged intermittent exercise, but the so-called meals were single foods which do not reflect the general practise in most sports [20], even though the results of performance agree with our findings. Present data cannot support the proposal that a LGI pre-exercise meal prior to football activity (in the time window utilised) improves performance due to an increase in fat oxidation and metabolism, and thereby possibly spare muscle glycogen.

The rationale for the use of LGI meals in sport and exercise is predicated on the purported shift in substrate utilisation and oxidation that occurs with a LGI meal providing a greater level of fat oxidation [36] and thus sparing muscle glycogen stores. Fat and $\mathrm{CHO}$ oxidation rates between LGI and HGI did not differ in the current study, which could reflect the intermittent high intensity protocol used to replicate the activity patterns seen in football. Where modifications in substrate oxidation are observed in other studies, they have commonly employed a continuous mode of exercise. Significant depletion of the body's muscle glycogen stores have been shown to occur during a football match $[3,26]$ due to the high intensity and duration of the sport. Researchers $[3,26]$ have reported the utilization of muscle glycogen by measuring pre and post levels and conclusively highlighted the need for high CHO meals. However, a recent investigation [20] also measured muscle glycogen levels between high GI and low GI foods in addition to a fasted control. They found a significantly greater muscle glycogen level following the $\mathrm{CHO}$ meals compared to the fasted control, but failed to measure any pre-meal or pre-exercise levels and therefore cannot report utilization between the high and low GI meals and the fasted control.

The insulinaemic and glucose response seen with the complete meals consumed in the current study is not entirely representative of the typical response expected with singular LGI and HGI foods. The response with the LGI meal is as expected with small elevations in serum insulin and blood glucose, with a return to baseline 30 min post meal that remains constant throughout the $3 \mathrm{~h}$ postprandial period. However, the HGI meal resulted in a greater, but non significant, rise in serum insulin with a concomitant significant elevation in post meal blood glucose increase, 

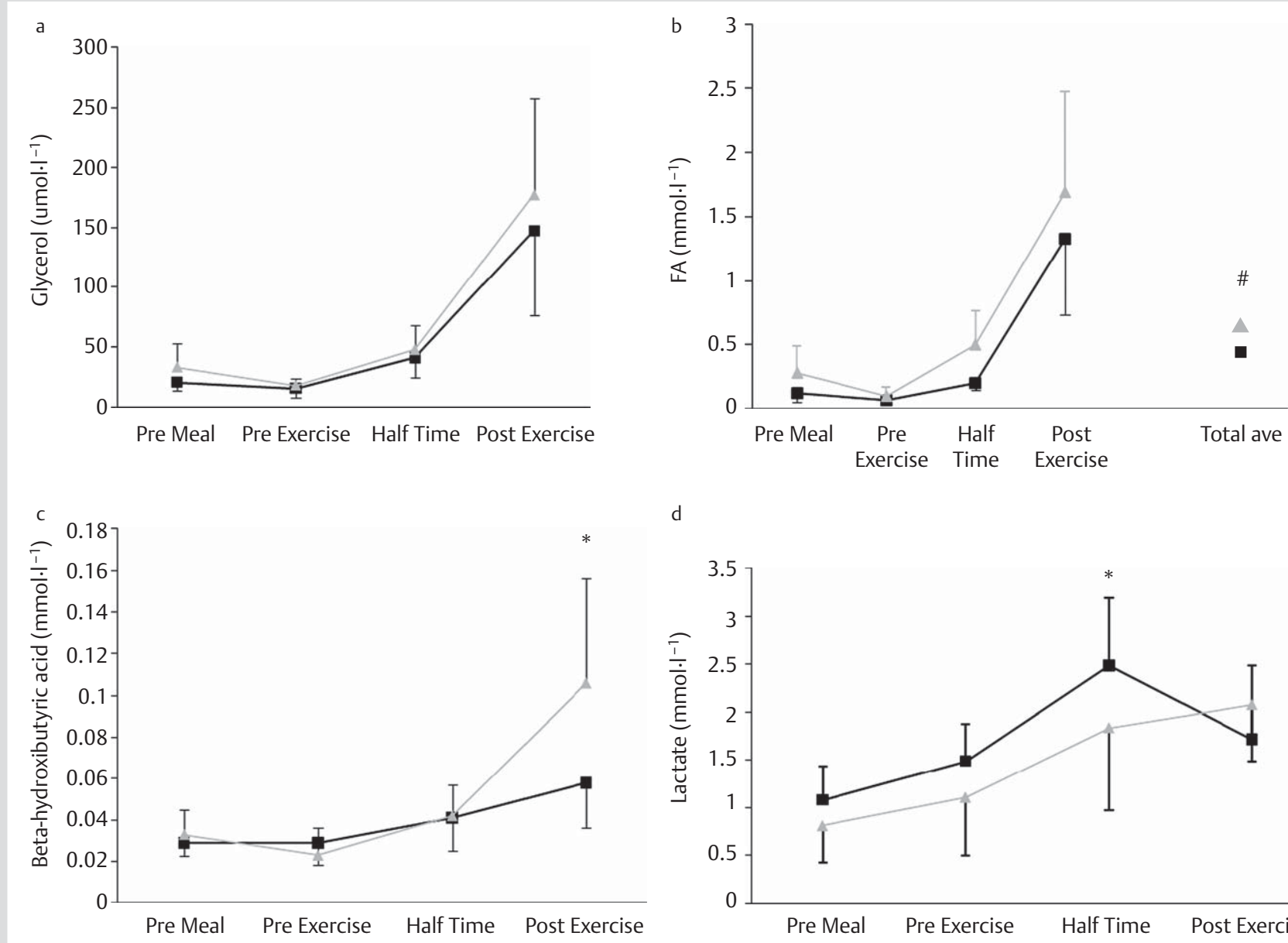

d

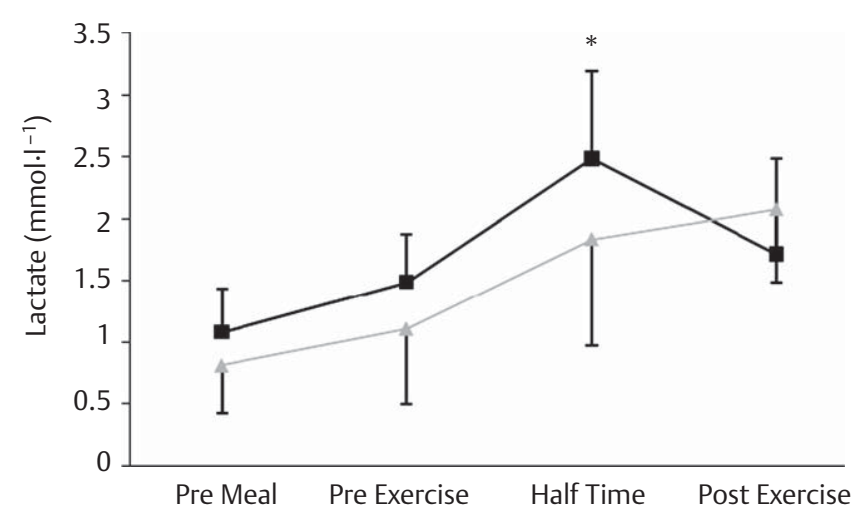

$\longrightarrow$ High Gl $\longrightarrow$ Low Gl

Fig. 3 a Glycerol, b NEFA, c $\beta$-hydroxybutyrate and $\mathbf{d}$ Lactate concentration prior to the test meal and throughout exercise. ${ }^{*}$ indicate where significant interactions occur.

\# indicate a significant condition effect
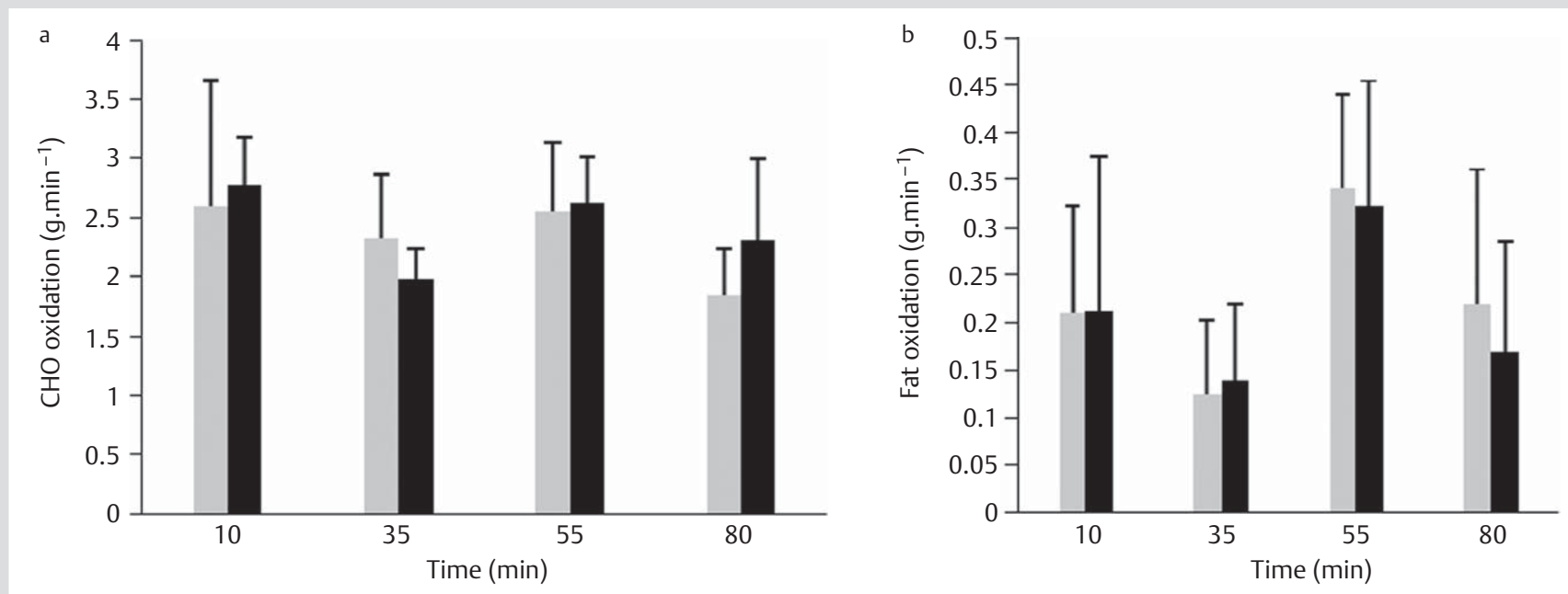

Low GI

High GI

Fig. 4 a CHO oxidation and b Fat oxidation measure during exercise. 

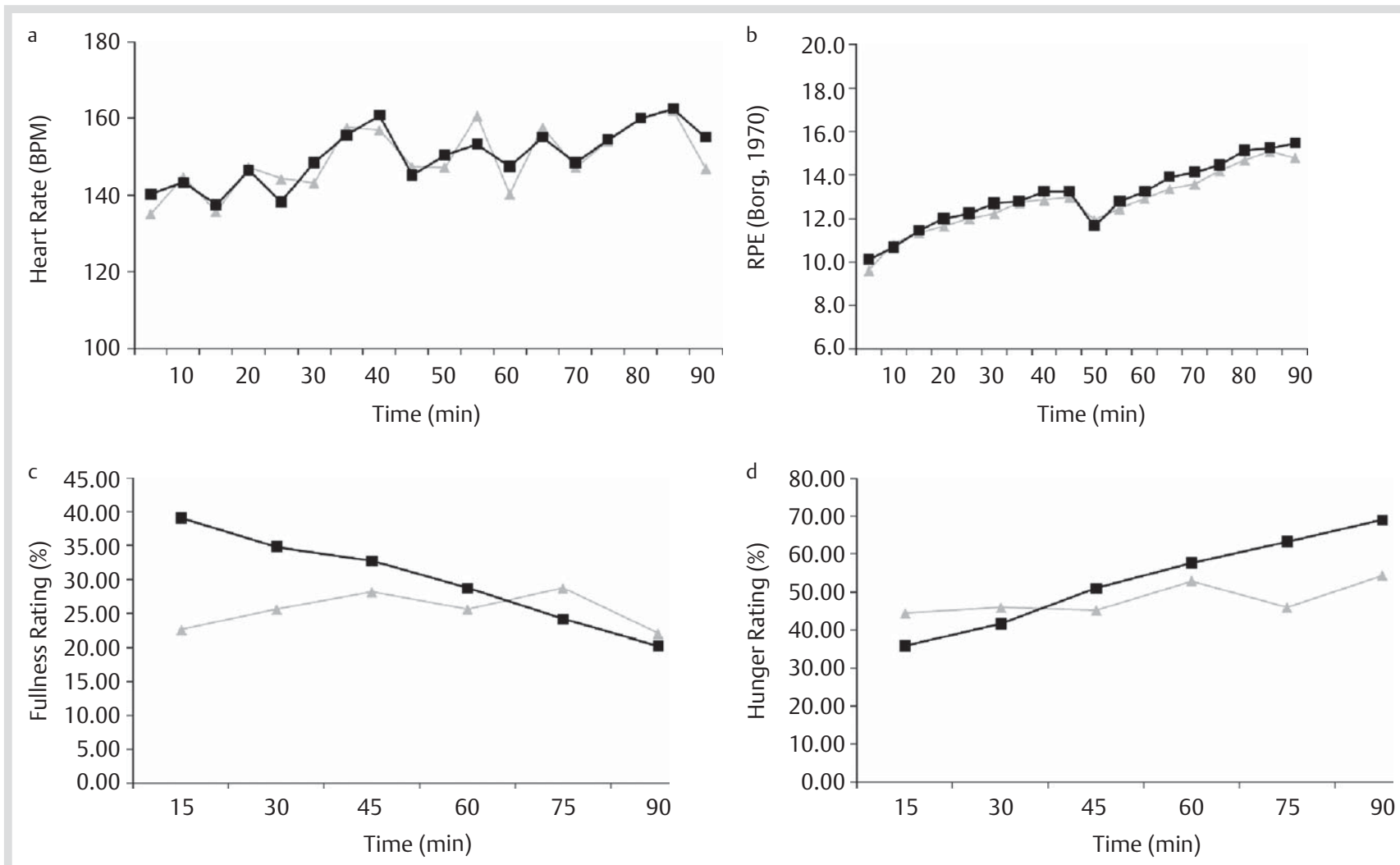

Fig. 5 a Heart rate, $\mathbf{b}$ RPE, $\mathbf{c}$ Fullness and $\mathbf{d}$ Hunger throughout exercise.

which then decreased throughout the postprandial period and the following exercise protocol with a slight rebound hypoglycaemia evident just before the onset of exercise. An increase in serum insulin may have been observed in the current study if further serum insulin analysis had occurred in the immediate postprandial period [30]. Similar glycaemic responses have been reported previously when comparing LGI and HGI foods prior to exercise $[11,30,36]$. The potential effects of rebound hypoglycaemia $[10,11,27,36]$ could prove detrimental to performance and metabolism in the early stages of competition and warrants further investigation.

Lower glucose and insulin responses that are attributed to the LGI meals have been suggested to promote satiety and suppress hunger [21]. However, the current study found no difference between meals for gut fullness or ratings of hunger. Furthermore, the glucose and insulin responses did not differ significantly during exercise. This may be due to the intermittent nature of the exercise or the inclusion of practical meals that contain protein as well as the $\mathrm{CHO}$, as protein catabolism can be insulinogenic. Throughout the exercise there was no evidence of hypoglycaemia at any stage, or even any evidence of hyperglycaemia at the end of the exercise between conditions. These findings concur with previous investigations [17] who even argue that the risk of reduced performance is minimal with preexercise $\mathrm{CHO}$ feeding despite relative large metabolic changes.

An increase in FA was observed towards the latter stages of the exercise bout, which was similar to previous reports $[2,18]$. This may be mediated by the frequent rest and low intensity exercise periods interspersed throughout the intermittent activity that allows for greater blood flow to the adipose tissue. Also the likely increase in epinephrine (with a concomitant decrease in insulin) supports the likelihood of an increase in lipolysis. Furthermore, the increase in FA is significantly augmented after a LGI meal compared to a HGI meal, probably as a consequence of the hyperglycaemic and hyperinsulinemic responses to the HGI meal in the postprandial period. Such findings have previously been observed [11] and suggest a persistent effect of hyperinsulinemia, even after insulin and glucose levels return to normal. The current study also found a significant interaction effect with $\beta$-hydroxybutyrate which indicates a greater ketosis at the end of the exercise.

The current study found a $2.78 \%$ faster completion of the $1 \mathrm{~km}$ run after LGI, and although not significant, 5 out of 8 participants were faster after LGI. This is similar to other studies that found no significant performance differences between conditions with tests measuring work done in a given period of time or set distance $[6,10,11,19,20,27]$. Only one study has found significant results in favour of a LGI meal prior to exercise with a $21 \mathrm{~km}$ time trial [35]. Most studies that have found differences between conditions $[8,32]$ have employed a time-to-exhaustion protocol which would not be seen in a sporting competition. In conclusion, the pre-exercise meal has no significant impact on performance after a $90 \mathrm{~min}$ intermittent high intensity exercise, suggesting that the type of $\mathrm{CHO}$ ingested does not radically alter substrate metabolism. However, the fact that 5 out of 8 participants provided faster times for the $1 \mathrm{~km}$ run warrants further investigation as does examination of muscle glycogen concentration post activity in order to provide an insight into likely substrate utilisation. 


\section{References}

1 Bangsbo J, Nørregaard L, Thorsøe F. The effect of carbohydrate diet on intermittent exercise performance. Int J Sports Med 1992; 13: 152-157

2 Bangsbo J. The physiology of soccer - with special reference to intense intermittent exercise. Acta Physiol Scand Suppl 1994; 151: 1-151

3 Bangsbo J, MohrM, Krustrup P. Physical and metabolic demands of training and match-play in the elite football player. J Sports Sci 2006; 24: 665-674

4 Bland JM, Altman DG. Multiple significance tests: the Bonferroni method. BMJ 1995; 310: 170

5 Borg G. Perceived exertion as an indicator of somatic stress. Scand J Rehabil Med 1970; 2: 93-98

6 Chen YJ, Wong SH, Wong CK, Lam CW, Huang YJ, Siu PM. Effect of preexercise meals with different glycemic indices and loads on metabolic responses and endurance running. Int J Sports Nutr Exerc Metab 2008; 18: 281-300

7 Clarke ND, Drust B, MacLaren DPM, Reilly T. Fluid provision and metabolic responses to soccer-specific exercise. Eur J Appl Physiol 2008; 104: 1069-1077

8 DeMarco HM, Sucher KP, Cisar CJ, Butterfield GE. Pre-exercise carbohydrate meals: application of glycemic index. Med Sci Sport Exerc 1999; 31: 164-170

9 Drust B, Reilly T, Cable NT. Physiological responses to laboratorybased soccer-specific intermittent and continuous exercise. J Sports Sci 2000; 18: 885-892

10 Febbraio MA, Stewart KL. CHO feeding before prolonged exercise: effect of glycemic index on muscle glycogenolysis and exercise performance. J Appl Physiol 1996; 81: 1115-1120

11 Febbraio MA, Keenan J, Angus DJ, Campbell SE, Garnham AP. Preexercise carbohydrate ingestion, glucose kinetics, and muscle glycogen use: effect of glycemic index. J Appl Physiol 2000; 89: 1845-1851

12 Foster-Powell $\mathrm{K}$, Holt SH, Brand-Miller JC. International table of glycemic index and glycemic load values. Am J Clin Nutr 2002; 76: 5-56

13 Frayn KN. Calculation of substrate oxidation rates in vivo from gaseous exchanges. J Appl Physiol 1983; 55: 628-634

14 Greenhaff PL, Nevill ME, Soderlund K, Bodin K, Boobis LH, Williams C, Hultman E. The metabolic responses of human type I and II muscle fibres during maximal treadmill sprinting. J Physiol 1994; 1: 149-155

15 Harriss DJ, Atkinson G. Update - Ethical standards in sport and exercise science research. Int J Sports Med 2011; 32: 819-821

16 Jenkins DJA, Wolever TMS, Taylor RH, Barker H, Fielden H, Baldwin JM, Bowling AC, Newman HC, Jenkins AL, GoffDV. Glycemic index of foods: a physiological basis for carbohydrate exchange. Am J Clin Nutr 1981; 34: 362-366

17 Jeukendrup AE, Killer $S C$. The myths surrounding pre-exercise carbohydrate feeding. Ann Nutr Metab 2010; 57 (Suppl 2): 18-25

18 Krustrup P, Mohr M, Steensberg A, Bencke J, Kær M, Bangsbo J. Muscle and blood metabolites during a soccer game: implications for sprint performance. Med Sci Sports Exerc 2006; 38: 1-10

19 Little JP, Chilibeck PD, Ciona D, Vandenberg A, Zello GA. The effects of low- and high-glycemic index foods on high intermittent exercise. Int J Sport Physiol Perform 2009; 4: 367-380
20 Little JP, Chilibeck PD, Ciona D, Forbes S, Rees H, Vandenberg A, Zello $G A$. Effect of low- and high-glycemic-index meals on metabolism and performance during high intermittent exercise. Int J Sports Nutr Exerc Metab 2010; 20: 447-456

21 Ludwig DS. The glycaemic index: physiological mechanisms related to obesity, diabetes, and cardiovascular disease. JAMA 2002; 287: 2414-2423

22 Moore LJS, Midgley AW, Thurlow S, Thomas G, Mc Naughton LR. Effect of the glycaemic index of a pre-exercise meal on metabolism and cycling time trial performance. J Sci Med Sport 2010; 13: 182-188

23 Neufer PD, Costill DL, Flynn MG, Kirwan JP, Mitchell JB, Houmard J. Improvements in exercise performance: effects of carbohydrate feedings and diet. J Appl Physiol 1987; 62: 983-988

24 Peden C, Sherman WM, D'Acquisto L, Wright DA. $1 \mathrm{~h}$ pre-exercise carbohydrate meals enhance performance. Med Sci Sports Exerc 1989; 2: S59

25 Reilly $T$, Thomas $V$. A motion analysis of work-rate in different positional roles in professional football match play. J Hum Mov Stud 1976; 2: 87-97

26 Saltin B. Metabolic fundamentals in exercise. Med Sci Sports Exerc 1973; 5: 137-146

27 Sparks MJ, Selig SS, Febbraio MA. Pre-exercise carbohydrate ingestion: effect of the glycemic index on endurance exercise performance. Med Sci Sport Exerc 1998; 30: 844-849

28 Stannard SR, Constantini NW, Brand Miller JC. The effect of glycemic index on plasma glucose and lactate levels during incremental exercise. Int J Sports Nutr Exerc Metab 2000; 10: 51-61

29 Stevenson EJ, Williams C, Nute L. The influence of the glycaemic index of breakfast and lunch on substrate utilisation during the postprandial periods and subsequent exercise. Br J Nutr 2005; 93: 885-893

30 Stevenson EJ, Williams C, Mash LE, Phillips B, Nute L. Influence of high-carbohydrate mixed meals with different glycemic indexes on substrate utilization during subsequent exercise in women. Am J Clin Nutr 2006; 84: 354-360

31 Stubbs J, Ferres S, Horgan G. Energy density of foods: effects on energy intake. Crit Rev Food Sci Nutr 2000; 40: 481-515

32 Thomas DE, Brotherhood JR, Brand JC. Carbohydrate feeding before exercise: effect of glycemic index. Int J Sports Med 1991; 12: 180-186

33 Wee SL, Williams C, Gray S, Horabin J. Influence of high and low glycemic index meals on endurance running capacity. Med Sci Spor Exerc 1999; 31: 393-399

34 Wolever TM, Jenkins DJ. The use of the glycemic index in predicting the blood glucose response to mixed meals. Am J Clin Nutr 1986; 43: $167-172$

35 Wong SHS, Siu PM, Lok A, Chen YJ, Morris J, Lam CW. Effect of the glycaemic index of pre-exercise carbohydrate meals on running performance. Eur J Sports Sci 2008; 8: 23-33

36 Wu C, Nicholas C, Williams C, Took A, Hardy L. The influence of highcarbohydrate meals with different glycaemic indices on substrate utilisation during subsequent exercise. Br J Nutr 2003; 90: 1049-1056 